\title{
Spatial Multizone Soundfield Reproduction: Theory and Design
}

\author{
Yan Jennifer Wu and Thushara D. Abhayapala, Senior Member, IEEE
}

\begin{abstract}
Spatial multizone soundfield reproduction over an extended region of open space is a complex and challenging problem in acoustic signal processing. In this paper, we provide a framework to recreate 2-D spatial multizone soundfields using a single array of loudspeakers which encompasses all spatial regions of interest. The reproduction is based on the derivation of an equivalent global soundfield consisting of a number of individual multizone soundfields. This is achieved by using spatial harmonic coefficients translation between coordinate systems. A multizone soundfield reproduction problem is then reduced to the reproduction over the entire region. An important advantage of this approach is the full use of the available dimensionality of the soundfield. This paper provides quantitative performances of a 2-D multizone system and reveals some fundamental limits on 2 -D multizone soundfield reproduction. The extensions of the multizone soundfield reproduction design in reverberant rooms are also included.
\end{abstract}

Index Terms-Ambisonics, cylindrical harmonic expansions, loudspeaker arrays, multizone, soundfield reproduction, spatial sound.

\section{INTRODUCTION}

$\mathbf{S}$ PATIAL multizone soundfield reproduction over an extended region of open space has recently drawn attention due to its various applications such as simultaneous car entertainment systems, surround sound systems in exhibition centers, personal loudspeaker systems in shared office space, and quiet zones in a noisy environment, where the aim is to provide listeners individual sound environment without physical isolated regions or using headphones. However, most of the existing research works in spatial soundfield reproduction mainly concentrated on a single zone, such as the Ambisonics [1]-[3], the Least Squares techniques [4]-[7], the wave field synthesis (WFS) approach [8]-[13], and the spherical harmonics-based systems [14]-[17]. Realization of multizone soundfield reproduction is a conceptually challenging problem and there is only a limited number of works reported in the literature.

Manuscript received December 02, 2009; revised September 13, 2010, November 12, 2010; accepted November 16, 2010. Date of publication December 17, 2010; date of current version June 01, 2011. The work of Y. J. $\mathrm{Wu}$ was supported in part by National ICT Australia. Preliminary work has been published in the 128th AES Convention, London, U.K., in 2010. Part of this work has appeared in the proceedings of International Conference of Acoustics, Speech, and Signal Processing (ICASSP) 2009. The associate editor coordinating the review of this manuscript and approving it for publication was Dr. Patrick A. Naylor.

The authors are with the Applied Signal Processing Group, School of Engineering, College of Engineering and Computer Science, The Australian National University, Canberra ACT 0200, Australia (e-mail: jennifer.wu@ @sise. anu.edu.au; thushara.abhayapala@anu.edu.au).

Color versions of one or more of the figures in this paper are available online at http://ieeexplore.ieee.org.

Digital Object Identifier 10.1109/TASL.2010.2097249
In 2007, I. Tashev, J. Droppo and M. Seltzer used a linear loudspeaker array consisting of 16 inexpensive speakers to demonstrate that sound waves cancel each other out in one area and become amplified in another. Someone stepping even a few paces to the side of the designated soundfield cannot hear the music [18]. This speaker array project, called "Personal Audio Space," was demonstrated in Microsoft Research TechFest 2007 [19]. The audio beamforming algorithm together with a sound localizer such as a camera or specialized microphone array is used in this project. However, the project is still in its early stages due to several technical challenging such as different hardware and software considerations for a wide range of frequencies, the extreme time precision requirement of the audio beamforming and the position tracking using image-processing techniques [20]. In 2008, Poletti proposed a 2-D multizone surround sound system using the least squares pressure matching approach [21]. It is considered the first published work in multizone soundfield reproduction; however, the investigations are mainly performed based on simulation results.

Motivated by these, we attempt to solve the height invariant (2-D) multizone problem by analyzing the problem with cylindrical harmonic expansions. In [22], we proposed a framework to recreate multiple 2-D soundfields at different locations within a single circular loudspeaker array. The key to this framework is to derive equivalent global multizone soundfield coefficients so that the spatial multizone soundfield reproduction problem can be reduced to the reproduction of a single soundfield over the entire region. This consists of two stages: 1) translating each individual desired soundfield to a single global coordinate system using the spatial harmonic coefficients translation theorem and 2) employing angular window functions to each soundfield to remove any inter-zone interference. Unfortunately, this second stage [(10) in [22]] is fundamentally incorrect since an angular windowed soundfield is not a physically realizable soundfield, i.e., it is not a valid solution to the wave equation.

Another method is introduced in [23] for a two-zone reproduction system. The method in [23] uses spatial band stop filters to suppress the unintended interzone interference in the regions of interests and pass the desired soundfields with no distortion. This was achieved by using the higher order spatial harmonics of one zone to cancel the undesirable effects of the lower order harmonics of the same zone on the other zones. However, this method can only be applicable for two zones.

In this paper, we propose a new method of finding an equivalent global soundfield coefficients that will produce all soundfield zones using the spatial harmonic coefficients translation theorem [22]-[24]. Specifically, we 1) translate the equivalent global soundfield coefficients into each soundfield zone and equate it to the desired soundfield coefficients of each 
zone, we then 2) construct simultaneous equations to obtain the equivalent global soundfield coefficients by the Least Squares approach, and 3) finally use any of the existing single zone soundfield reproduction techniques to reproduce the desired multizones using an array of loudspeakers. Further, we have revealed some fundamental limits on 2-D multizone soundfield reproduction.

We also extend the multizone soundfield reproduction design to reverberant rooms by applying the reverberant field design proposed in [15]. Simulations results show favorable reproduction performance.

The paper is organized as follows. In Section II, we properly formulate the problem for multizone soundfield reproduction. In Section III, we present a novel approach to find the equivalent global soundfield coefficients by the spatial harmonic coefficients translation theorem. In Section IV, we illustrate the loudspeaker design to reproduce the desired multizones by the continuous loudspeaker method and the Least Squares method using a single loudspeaker array. Some of the fundamental limits of the multizone system are revealed in Section V. In Section VI, we extend the multizone soundfield reproduction in reverberant environments. Finally, we present the simulations results to verify the multizone system performances.

Notation: Throughout this paper, we use the following notations: matrices and vectors are represented by upper and lower bold face respectively, e.g., $\boldsymbol{T}$ and $\boldsymbol{\alpha}$. A unit vector in the direction $\boldsymbol{x}$ is denoted by $\hat{\boldsymbol{x}}$, i.e., $\hat{\boldsymbol{x}}=\boldsymbol{x} /\|\boldsymbol{x}\|$, where $\|\cdot\|$ denotes the Euclidean distance $(\|x\|=\sqrt{\boldsymbol{x} \cdot \boldsymbol{x}})$ and "." is used to represent the inner product of two vectors. The vector length is denoted by $x$, i.e., $\boldsymbol{x}=x \hat{\boldsymbol{x}}$ or $x=\|\boldsymbol{x}\|$. The imaginary unit is denoted by $i(=\sqrt{-1})$. The superscripts " $d$ " and "a" are used to represent the desired soundfield and the actual soundfield respectively.

\section{PROBLEM FORMULATION}

\section{A. Multizone Soundfield Model}

We assume that there are $Q$ nonoverlapping 2-D spatial zones and corresponding desired spatial soundfields. As shown in Fig. 1, the radius and the origin of the $q$ th spatial zone are denoted as $R_{z}^{(q)}$ and $\mathcal{O}_{q}$, respectively, where $\mathcal{O}_{q}$ is located at the polar coordinate $\left(r^{(q 0)}, \theta^{(q 0)}\right)$ with respect to a global origin $\mathcal{O}$. Any arbitrary observation point within this circular $q$ th spatial zone is denoted as $\left(R^{(q)}, \Omega^{(q)}\right)$, which is $(r, \theta)$ with respect to the global origin $\mathcal{O}$. The loudspeakers are placed on a circle with radius $R_{p} \geq r$ from $\mathcal{O} .{ }^{1}$ The loudspeaker weight at angle $\phi$ is denoted as $\rho_{p}(\phi, k)$, where $k=2 \pi f / c$ is the wavenumber, $f$ is the frequency and $c$ is the speed of sound propagation. In general case, we assume $c$ is $340 \mathrm{~m} / \mathrm{s}$ in our simulations. Throughout this paper, we use $k$ instead of $f$ to represent frequency since we assume constant $c$. We assume no sound sources or scattering objects being present inside the reproduction area.

${ }^{1}$ Circular loudspeaker array is not a requirement for the loudspeaker configuration as long as the loudspeakers are placed on or outside the circle of radius $R_{p}$; however, for simplicity, we use circular loudspeaker array in this paper.

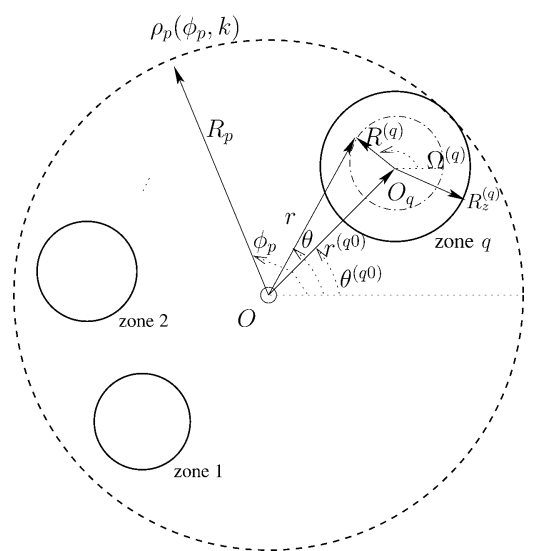

Fig. 1. Geometry of the multizone sound reproduction system. The radius and the origin of the $q$ th spatial zone are denoted as $R_{z}^{(q)}$ and $\mathcal{O}_{q}$, respectively, where $\mathcal{O}_{q}$ is located at the polar coordinate $\left(r^{(q 0)}, \theta^{(q 0)}\right)$ with respect to a global origin $\mathcal{O}$. Any arbitrary observation point within this circular spatial zone is denoted as $\left(R^{(q)}, \Omega^{(q)}\right)$, which is $(r, \theta)$ with respect to the global origin $\mathcal{O}$. The loudspeakers are placed on a circle with radius $R_{p} \geq r$ from $\mathcal{O}$. The loudspeaker weight at angle $\phi_{p}$ is denoted as $\rho_{p}\left(\phi_{p}, k\right)$.

\section{B. Cylindrical Harmonic Expansion of a Soundfield}

As shown in Fig. 1, any arbitrary 2-D (height invariant) desired soundfield of the $q$ th spatial zone, $S^{\mathrm{d}(q)}\left(R^{(q)}, \Omega^{(q)} ; k\right)$ can be represented by the following cylindrical harmonic expansion representation [16], [17]:

$$
S^{\mathrm{d}(q)}\left(R^{(q)}, \Omega^{(q)} ; k\right)=\sum_{m=-\infty}^{\infty} \alpha_{m}^{\mathrm{d}(q)}(k) J_{m}\left(k R^{(q)}\right) e^{i m \Omega^{(q)}}
$$

where $J_{m}(\cdot)$ are the Bessel functions of order $m, \alpha_{m}^{\mathrm{d}(q)}(k)$ are a set of coefficients uniquely representing the $q$ th desired soundfield and the superscript "d" represents the desired soundfield. Note, the representation (1) is in the form of a Fourier series expansion. Any arbitrary 2-D (height invariant) soundfield due to any number of cylindrical waves and/or plane waves can be represented by (1).

\section{Mode Limitedness}

The representation (1) has an infinite number of orthogonal modes; however, we can truncate this series expansion to a finite number within the region of interest due to the properties of the Bessel functions (see Fig. 2) and the fact that the soundfield has to be bounded within a spatial region where all sources are outside [25], [26]. Hence, we can truncate (1) to

$$
S^{\mathrm{d}(q)}\left(R^{(q)}, \Omega^{(q)} ; k\right)=\sum_{m=-M_{q}}^{M_{q}} \alpha_{m}^{\mathrm{d}(q)}(k) J_{m}\left(k R^{(q)}\right) e^{i m \Omega^{(q)}}
$$

where the desired soundfield of the $q$ th spatial zone is mode limited to $M_{q}$ (That is, the desired sound field is entirely described by $2 M_{q}+1$ lowest modes)

$$
M_{q}=\left\lceil k e R_{z}^{(q)} / 2\right\rceil
$$

with error in truncation is less than $16.1 \%$ [25]. 


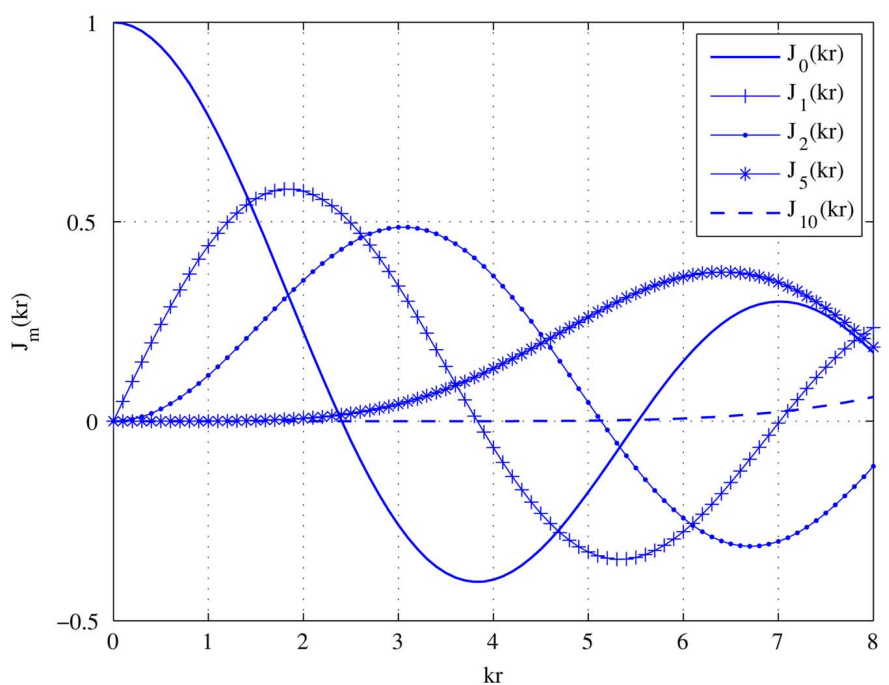

Fig. 2. Properties of the Bessel functions for order $0,1,2$, 5, 10, i.e., $J_{0}(k r), J_{1}(k r), J_{2}(k r), J_{5}(k r)$ and $J_{10}(k r)$.

\section{Equivalent Global Soundfield}

We seek to find an equivalent global soundfield which consists of $Q$ nonoverlapping individual multizone soundfields. Thus, the problem of reproduction of multiple spatial soundfields can be reduced to reproduction of the global desired soundfield over the entire region. That is a single-zone reproduction problem, which has been well addressed before [5], [8], [13], [14], [17], [15].

Let the set of coefficients $\left\{\beta_{m}^{\mathrm{d}}(k)\right\}$ uniquely represent the desired global soundfield that will produce all soundfield zones. We express the desired global soundfield using cylindrical harmonic expansion as [16], [17]

$$
S^{\mathrm{d}}(r, \theta ; k)=\sum_{m=-M_{0}}^{M_{0}} \beta_{m}^{\mathrm{d}}(k) J_{m}(k r) e^{i m \theta}
$$

where $\beta_{m}^{\mathrm{d}}(k)$ are mode limited to $M_{0}=\left\lceil k e R_{p} / 2\right\rceil$ and $R_{p}$ is the radius of the smallest circle that enclose all spatial zones of interest.

In the following subsections, we will relate the desired soundfield coefficients of individual zones $\alpha_{m}^{\mathrm{d}(q)}(k)$ to the equivalent global soundfield coefficients $\beta_{m}^{\mathrm{d}}(k)$.

\section{E. Spatial Harmonic Coefficient Translation}

Consider a soundfield within a source free region of space. Let $\mathcal{O}_{1}$ and $\mathcal{O}_{2}$ be the origins of two coordinate systems that have the same orientation but are displaced with a known translation (see Fig. 3). Let $\left(r^{(12)}, \theta^{(12)}\right)$ be the coordinates of $\mathcal{O}_{2}$ with respect to $\mathcal{O}_{1}$. Also let $\left\{\alpha_{m}^{(1)}(k)\right\}$ and $\left\{\alpha_{m}^{(2)}(k)\right\}$ be the sets of coefficients of the soundfield within the source free region with respect to the two coordinate systems, respectively. Now, we state the following translation theorem: ${ }^{2}$

Theorem 1: The soundfield coefficients $\alpha_{m}^{(1)}(k)$ and $\alpha_{m}^{(2)}(k)$ are related by

$$
\alpha_{m}^{(1)}(k)=\alpha_{m}^{(2)}(k) * T_{m}^{(21)}\left(r^{(12)}, \theta^{(12)} ; k\right)
$$

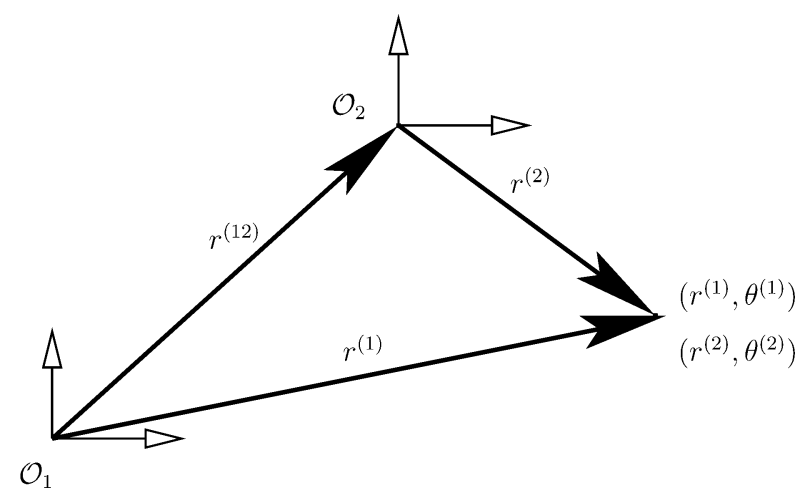

Fig. 3. Geometry related to Translation Theorem 1. A point within in a source free region with coordinates $\left(r^{(1)}, \theta^{(1)}\right)$ and $\left(r^{(2)}, \theta^{(2)}\right)$ is shown with respect to two origins $\mathcal{O}_{1}$ and $\mathcal{O}_{2}$, respectively. The coordinate of the origin $\mathcal{O}_{2}$ with respect to the origin $\mathcal{O}_{1}$ is given by $\left(r^{(12)}, \theta^{(12)}\right)$. Similarly, the coordinate of the origin $\mathcal{O}_{1}$ with respect to the origin $\mathcal{O}_{2}$ can be defined as $\left(r^{(21)}, \theta^{(21)}\right)$.

where

$$
T_{m}^{(21)}\left(r^{(12)}, \theta^{(12)} ; k\right) \triangleq J_{m}\left(k r^{(12)}\right) e^{-i m \theta^{(12)}}
$$

and " $*$ " denotes the discrete convolution with mode order $m$.

The proof is given in Appendix 1.

We refer $T_{m}^{(21)}$ as the translation operator from the origin $\mathcal{O}_{2}$ to $\mathcal{O}_{1}$. Then, $T_{m}^{(12)}$ is the translation operator from the origin $\mathcal{O}_{1}$ to $\mathcal{O}_{2}$. We have the following theorem.

\section{Theorem 2:}

$$
T_{m}^{(12)}\left(r^{(21)}, \theta^{(21)}\right)=T_{m}^{(21)}\left(r^{(12)}, \theta^{(12)}+\pi\right) .
$$

The proof is given in Appendix 2.

\section{F. Problem Setup}

By applying the spatial harmonic coefficient translation theorem, we can translate the equivalent global soundfield into each individual zone. Thus, the problem we consider in this paper is as follows.

Given the desired soundfields of $Q$ spatial zones by a set of finite number of coefficients $\left\{\alpha_{m}^{\mathrm{d}(q)}(k)\right\}$, how can we find the equivalent global soundfield coefficients $\beta_{m}^{\mathrm{d}}(k)$ such that

$$
\begin{aligned}
& \beta_{m}^{\mathrm{d}}(k) * T_{m}^{(0 q)}=\alpha_{m}^{\mathrm{d}(q)}(k), \\
& \text { for } q=1, \ldots, Q \text { and } m=-M_{q}, \ldots, M_{q}
\end{aligned}
$$

where $T_{m}^{(0 q)}$ is the translation operator from the global coordinate system to the coordinate system of the $q$ th spatial zone.

\section{FINDING THE GLOBAL SOUNDFIELD COEFFICIENTS}

We write the convolution in (8) as a summation to obtain

$$
\sum_{n=-M_{0}}^{M_{0}} \beta_{n}^{\mathrm{d}}(k) T_{m-n}^{(0 q)}=\alpha_{m}^{\mathrm{d}(q)} .
$$

Now, we write (9) for $q=1, \ldots, Q$ and construct $Q$ simultaneous equations, which we express in matrix form as

$$
\boldsymbol{\alpha}^{\mathrm{d}}(k)=\boldsymbol{T}(k) \boldsymbol{\beta}^{\mathrm{d}}(k)
$$


where

$$
\begin{aligned}
\boldsymbol{\beta}^{\mathrm{d}}(k) \triangleq & {\left[\beta_{-M_{0}}^{\mathrm{d}}(k), \ldots, \beta_{M_{0}}^{\mathrm{d}}(k)\right]^{T} } \\
\boldsymbol{\alpha}^{\mathrm{d}}(k) \triangleq & {\left[\alpha_{-M_{1}}^{\mathrm{d}(1)}(k), \ldots, \alpha_{M_{1}}^{\mathrm{d}(1)}(k), \alpha_{-M_{2}}^{\mathrm{d}(2)}(k), \ldots,\right.} \\
& \left.\alpha_{M_{2}}^{\mathrm{d}(2)}(k), \ldots, \alpha_{-M_{q}}^{\mathrm{d}(q)}(k), \ldots, \alpha_{M_{q}}^{\mathrm{d}(q)}\right]^{T}
\end{aligned}
$$

and

$$
\boldsymbol{T}(k) \triangleq\left(\begin{array}{ccc}
T_{-M_{1}+M_{0}}^{(01)} & \cdots & T_{-M_{1}-M_{0}}^{(01)} \\
\vdots & \ddots & \vdots \\
T_{M_{1}+M_{0}}^{(01)} & \cdots & T_{M_{1}-M_{0}}^{(01)} \\
T_{-M_{2}+M_{0}}^{(02)} & \cdots & T_{-M_{2}-M_{0}}^{(02)} \\
\vdots & \ddots & \vdots \\
T_{M_{2}+M_{0}}^{(02)} & \cdots & T_{M_{2}-M_{0}}^{(02)} \\
\vdots & \vdots & \vdots \\
T_{-M_{q}+M_{0}}^{(0 q)} & \cdots & T_{-M_{q}-M_{0}}^{(0 q)} \\
\vdots & \ddots & \vdots \\
T_{M_{q}+M_{0}}^{(0 q)} & \cdots & T_{M_{q}-M_{0}}^{(0 q)}
\end{array}\right) .
$$

We use the Least Squares method to solve (10) and obtain the global soundfield coefficients

$$
\boldsymbol{\beta}^{\mathrm{d}}(k)=\boldsymbol{T}(k)^{\dagger} \boldsymbol{\alpha}^{\mathrm{d}}(k)
$$

where $\boldsymbol{T}(k)^{\dagger}=\left[\boldsymbol{T}(k)^{H} \boldsymbol{T}(k)\right]^{-1} \boldsymbol{T}(k)^{H}$ is the Moore-Penrose (Pseudo) Inverse of $\boldsymbol{T}(k)$.

We have the following comments.

- The equivalent global soundfield coefficients $\beta_{m}^{\mathrm{d}}(k)$ are expressed as a function of the desired individual zone coefficients and the translation coefficients of origins of each zone.

- Equation (14) involves a matrix inversion, hence the robustness of the solution is dependent on the eigenvalues of $\boldsymbol{T}(k)^{H} \boldsymbol{T}(k)$, i.e., the squared singular values of $\boldsymbol{T}(k)$, which is a function of the zone positions $\left(r^{(0 q)}, \theta^{(0 q)}\right)$. Thus, each zone needs to be carefully positioned to avoid the poorly conditioned inverse problem [29].

- The robustness of the solution can be improved by introducing well known regularization techniques such as Tikhonov method. In the single-zone soundfield reproduction literature, the Tikhonov regularization was used successfully in [4], [29].

\section{LOUdSPEAKER WeIGHTS DESIGN FOR Multizone SOUNDFIELD REPRODUCTION}

Knowing the desired soundfield coefficients for the global region, the multizone soundfield reproduction problem is now reduced to reproduction of the desired soundfield (4) over the entire region. It does not necessarily require a circular loudspeaker array to reproduce the soundfield and the system could be extended to a general loudspeaker setting. However, for simplicity, a circular loudspeaker array is used to reproduce the multizone soundfield. As indicated in Fig. 1, we assume that the loudspeakers are placed on a circle with radius $R_{p} \geq r$ from the global origin $\mathcal{O}$, where the loudspeaker weight at angle $\phi_{p}$ is denoted as $\rho_{p}\left(\phi_{p}, k\right)$. We illustrate the loudspeaker design using the continuous loudspeaker method and the Least Squares method, respectively, in the following section.

\section{A. Soundfield Reproduction Using Continuous Loudspeaker Method}

In the continuous loudspeaker method, the underlying structure of the loudspeaker weights is a function of the desired soundfield, the loudspeaker position and the frequency [17]. By using the desired sound coefficients for the entire soundfield $\beta_{m}^{\mathrm{d}}(k)$, we can use $P$ discrete loudspeakers equally $\Delta \phi$ spaced on a circle of radius $R_{p}$, provided $P>2 M_{0}$. The loudspeaker weights are then given by [17]

$$
\rho_{p}\left(\phi_{p}, k\right)=\sum_{m=-M_{0}}^{M_{0}} \frac{2}{i \pi H_{m}^{(1)}\left(k R_{p}\right)} \beta_{m}^{\mathrm{d}}(k) e^{i m \phi_{p}} \Delta \phi
$$

where $H_{m}^{(1)}(\cdot)$ is the $m$ th-order Hankel function of the first kind.

The corresponding reproduced soundfield can be obtained by

$$
\begin{aligned}
& S^{\mathrm{a}}\left(R^{(q)}, \Omega^{(q)} ; k\right) \\
& \quad=\sum_{p=1}^{P} \rho_{p}\left(\phi_{p}, k\right) \frac{i}{4} H_{0}^{(1)}\left(k\left\|R_{p} \hat{\boldsymbol{\phi}}_{p}-R^{(q)} \hat{\boldsymbol{\Omega}}^{(q)}\right\|\right)
\end{aligned}
$$

where the unit position vectors $\hat{\boldsymbol{\phi}}_{p} \equiv\left(1, \phi_{p}\right)$ and $\hat{\boldsymbol{\Omega}}^{(q)} \equiv$ $\left(1, \Omega^{(q)}\right)$.

\section{B. Soundfield Reproduction Using Least Squares Method}

We can also use Least Squares method to calculate the loudspeaker weights. We define the loudspeaker weights vector $\boldsymbol{\rho}(k)$ as $\rho(k)=\left[\rho_{1}(k), \ldots, \rho_{P}(k)\right]^{T}$, and it becomes [16]

$$
\boldsymbol{\rho}(k)=\boldsymbol{H}(k)^{\dagger} \boldsymbol{\beta}^{\mathrm{d}}(k)
$$

where $\boldsymbol{H}(k)^{\dagger}$ is the pseudo inverse of $\boldsymbol{H}(k), \boldsymbol{\beta}^{\mathrm{d}}(k)=$ $\left[\beta_{-M_{0}}^{\mathrm{d}}(k), \ldots, \beta_{M_{0}}^{\mathrm{d}}\right]^{T}$ and

$\boldsymbol{H}(k)=\frac{i}{4}\left(\begin{array}{ccc}H_{-M}^{(1)}\left(k R_{p}\right) e^{i M \phi_{1}} & \cdots & H_{-M}^{(1)}\left(k R_{p}\right) e^{i M \phi_{P}} \\ \vdots & \ddots & \vdots \\ H_{M}^{(1)}\left(k R_{p}\right) e^{-i M \phi_{1}} & \cdots & H_{M}^{(1)}\left(k R_{p}\right) e^{-i M \phi_{P}}\end{array}\right)$.

The corresponding reproduced soundfield is given by (16).

Besides the above two methods, the Wave Field Synthesis (WFS) technique may also be used to reconstruct the required global soundfield by either implementing (16) as a soundfield consist of $P$ nearfield sources or by decomposition the required global soundfield into an equivalent set of plane wave sources.

\section{Fundamental Limits on Multizone SOUNDFIELD REPRODUCTION}

According to the theory of (single-zone) spatial soundfield reconstruction [17], a soundfield within a circular region of radius $R_{p}$ can be approximately described by $2 M_{0}+1$ soundfield coefficients, where $M_{0}=\left\lceil k \exp (1) R_{p} / 2\right\rceil$ [25]. The maximum possible error in this approximation is shown to be $16 \%$ [25], with the error exponentially decaying with addition of extra coefficients. This fundamental property of wavefields ${ }^{3}$ is termed as mode limitedness of an arbitrary soundfield within a finite region of space and the number $M_{0}$ is termed as the dimensionality [25] of the region.

\footnotetext{
${ }^{3}$ This is valid for Electro-magnetic wavefields as well
} 
Thus, to reproduce a given soundfield within a spatial region of radius of $R_{p}$ with maximum error of $16 \%$, we need at least $2 M_{0}+1$ loudspeakers. In the case of multizone soundfield reproduction, each soundfield is mode limited to $M_{q}=\left\lceil k e R_{z}^{(q)}\right\rceil$, for $q=1, \ldots, Q$. Hence, for a nonoverlaping $Q$ multizones with mode limitedness of $M_{q}$, where $M_{q}=\left\lceil k e R_{q} / 2\right\rceil$ and $R_{q}$ is the radius of the $q$ th zone, the total mode limitedness is given by $M_{1}+M_{2}+\cdots+M_{Q}$. If all multizones are confined to a circular region of radius $R_{p}$ with mode limitedness of $M_{0}$, then we need

$$
M_{0} \geq\left(M_{1}+M_{2}+\ldots+M_{Q}\right)
$$

in order to reproduce all multizones. This result fundamentally governs the limit of spatial multizone sound reproduction. The ability of multizone reproduction is dependent on the total number of modes required from each zone as well as the total number of modes available from the enclosed region. Also note that this result is dependent on relevant radii and the frequency of operation.

\section{EXTENSION TO REPRODUCTION IN REVERBERANT ROOMS}

In previous sections, we have assumed that the soundfield reproduction is performed in free field environments, this is not feasible in practice. Nevertheless, we could easily extend the multizone soundfield reproduction method introduced in this paper to work in reverberant rooms by applying the reverberant field design proposed in [15]. Specifically, the [15] shows how to reproduce a given soundfield in the form of (4) within a reverberant environment. Since we reduce the multizone reproduction problem to a single-zone soundfield in the form of (4), it is straightforward to use the technique in [15]. Here we briefly outline this technique, which we use in our simulation in Section VII.

Let $\tilde{H}_{p}(r, \theta ; k)$ be the room acoustic transfer function between the $p$ th loudspeaker and a point $(r, \theta)$. Then the reproduced soundfield from all $P$ loudspeakers is given by

$$
S^{\mathrm{a}}(r, \theta ; k)=\sum_{p=1}^{P} \rho_{p}(k) \tilde{H}_{p}(r, \theta ; k)
$$

where $\rho_{p}(k)$ is the frequency dependent weight of the $p$ th loudspeaker.

The acoustic transfer function $\tilde{H}_{p}(r, \theta ; k)$ can be viewed as the received signal at a point $(r, \theta)$ due to a unit-amplitude signal (impulse) from the $p$ th loudspeaker. Hence, it can be written in the form of (1)

$$
\tilde{H}_{p}(r, \theta ; k)=\sum_{m=-\infty}^{\infty} \gamma_{m}(p, k) J_{m}(k r) e^{i m \theta}
$$

where $\gamma_{m}(p, k)$ are the soundfield coefficients of the room response [15] for the $p$ th loudspeaker.
By substituting (21) into (20) and equating to the desired global soundfield given in (4), we can derive the loudspeaker weight vector $\boldsymbol{\rho}(k)$ in a reverberant room as

$$
\boldsymbol{\rho}(k)=\boldsymbol{A}(k)^{\dagger} \boldsymbol{\beta}^{\mathrm{d}}(k)
$$

where $\boldsymbol{A}(k)^{\dagger}=\left(\boldsymbol{A}^{H}(k) \boldsymbol{A}(k)\right)^{-1} \boldsymbol{A}^{H}(k)$ is the pseudo inverse of the matrix of the coefficients of the room response of all loudspeakers

$A=\left(\begin{array}{cccc}\gamma_{-M_{0}}(1, k), & \gamma_{-M_{0}}(2, k) & \cdots & \gamma_{-M_{0}}(P, k) \\ \gamma_{-M_{0}+1}(1, k), & \gamma_{-M_{0}+1}(2, k) & \cdots & \gamma_{-M_{0}+1}(P, k) \\ \vdots & \vdots & \ddots & \vdots \\ \gamma_{M_{0}}(1, k), & \gamma_{M_{0}}(2, k) & \cdots & \gamma_{M_{0}}(P, k)\end{array}\right)$

and $\beta^{\mathrm{d}}(k)$ is the vector of desired global soundfield coefficients defined before. In [15], additional weighting factor was introduced to scale modes differently. In this case $\boldsymbol{A}(k)^{\dagger}$ in (22) is given by $\boldsymbol{A}(k)^{\dagger}=\left(\boldsymbol{A}^{H}(k) \boldsymbol{W} \boldsymbol{A}(k)\right)^{-1} \boldsymbol{A}^{H}(k) \boldsymbol{W}$, where $\boldsymbol{W}$ is a diagonal matrix with diagonal elements given by $\int_{0}^{k R_{p}}\left[J_{m}(k r)\right]^{2} r d r$ which is proportional to the total energy associated with the mode $m$ within the entire reproduction region.

Note that we need to know the room response coefficients of each loudspeakers to apply this method. Normally, these are measured by microphone recordings (see [15] for detail). We use (22) to calculate the loudspeaker weights in Section VII-B and VII-C to verify the performances of the proposed multizone soundfield reproduction system in reverberant rooms.

\section{SimULATION}

\section{A. Two-Zone Example in Free Field}

In the first example, we consider two circular reproduction zones of radii $0.5 \mathrm{~m}$ which are in-line with each other. Zone 1 and Zone 2 are both $1.0 \mathrm{~m}$ away from the global origin $\mathcal{O}$. The desired soundfields consist of 50 random plane waves at $1000 \mathrm{~Hz}$. We use random plane waves to avoid the system being affected by the incoming wave source directions. In this example, we equally place 57 loudspeakers on a circle of $1.5 \mathrm{~m}$ while we calculate loudspeaker weights using the continuous method. The resulting reproduced field is shown in Fig. 4. The top two plots show the real and imaginary parts of the desired two-zone soundfield, and the bottom two plots show the soundfield reproduced by the loudspeaker array. The reproduced two-zone soundfield corresponds well to the desired multizone soundfield where the zone boundaries of the two reproduction regions are indicated in two circles. We define the mean squared reproduction error in the $q$ th zone as shown in (24) at the bottom of the page, which is a function of the radius $R_{z}^{(q)}$ of the zone and the frequency. The mean squared reproduction error in this example is $0.51 \%$, which indicates a very good reproduction.

$$
\epsilon^{(q)}\left(R_{z}^{(q)} ; k\right) \triangleq \frac{\int_{0}^{R_{z}^{(q)}} \int_{0}^{2 \pi}\left|S^{\mathrm{d}(q)}\left(R^{(q)}, \Omega^{(q)} ; k\right)-S^{\mathrm{a}(q)}\left(R^{(q)}, \Omega^{(q)} ; k\right)\right|^{2} d \Omega^{(q)} d R^{(q)}}{\int_{0}^{R_{z}^{(q)}} \int_{0}^{2 \pi}\left|S^{\mathrm{d}(q)}\left(R^{(q)}, \Omega^{(q)} ; k\right)\right|^{2} d \Omega^{(q)} d R^{(q)}}
$$



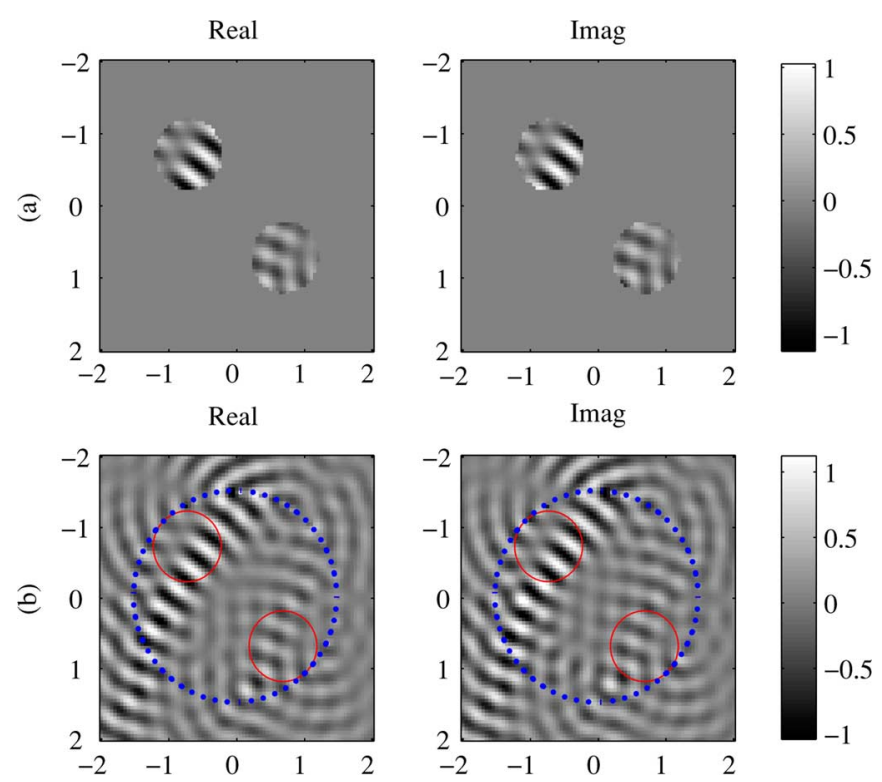

Fig. 4. Reproduction of a 2-D two-zone soundfield with radius $0.5 \mathrm{~m}$ each in free field. The desired soundfields consist of 50 random plane waves at $1000 \mathrm{~Hz}$. Two zones are located at $\theta^{(10)}=135^{\circ}, r^{(10)}=1 \mathrm{~m}$ and $\theta^{(20)}=-45^{\circ}, r^{(20)}=1 \mathrm{~m}$, respectively. (a) Desired field, and (b) reproduced field. We have equally placed 57 loudspeakers on a circle of $R=1.5 \mathrm{~m}$, where the loudspeaker positions are indicated in the dotted circle. In this case, the reproduced error calculated based on (24) is $0.51 \%$.

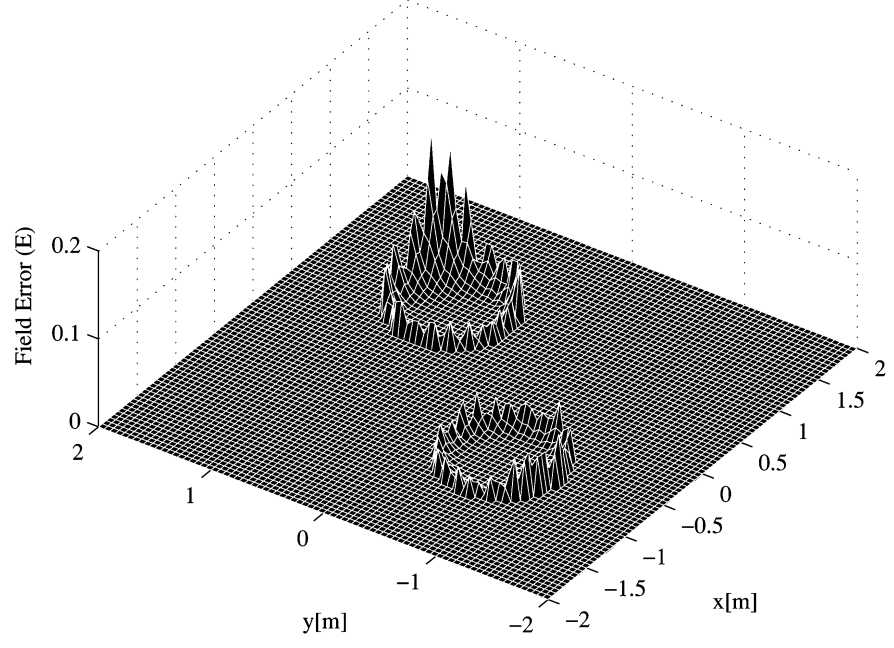

Fig. 5. Actual reproduced field error $\mathrm{E}\left(R^{(q)}, \Omega^{(q)} ; k\right)$ defined in (25) of a 2-D two-zone soundfield with radius $0.5 \mathrm{~m}$ each in free field. The desired soundfields consist of 50 random plane waves at $1000 \mathrm{~Hz}$. Two zones are located at $\theta^{(10)}=$ $135^{\circ}, r^{(10)}=1 \mathrm{~m}$ and $\theta^{(20)}=-45^{\circ}, r^{(20)}=1 \mathrm{~m}$, respectively. We have equally placed 57 loudspeakers on a circle of $R=1.5 \mathrm{~m}$. For demonstration purpose, we only plot the actual reproduced field error at each point in the zones of interest.

Fig. 5 depicts the actual reproduced field error at each point inside the zones of interest where the error is defined as

$$
\begin{aligned}
& \mathrm{E}\left(R^{(q)}, \Omega^{(q)} ; k\right) \\
& \triangleq \frac{\left|S^{\mathrm{d}(q)}\left(R^{(q)}, \Omega^{(q)} ; k\right)-S^{\mathrm{a}(q)}\left(R^{(q)}, \Omega^{(q)} ; k\right)\right|}{S^{\mathrm{d}(q)}\left(R^{(q)}, \Omega^{(q)} ; k\right)}
\end{aligned}
$$

where $S^{\mathrm{a}(q)}\left(R^{(q)}, \Omega^{(q)} ; k\right)$ denotes the actual reproduced soundfield at point $\left(R^{(q)}, \Omega^{(q)}\right)$ inside the $q$ th zone. For demon-

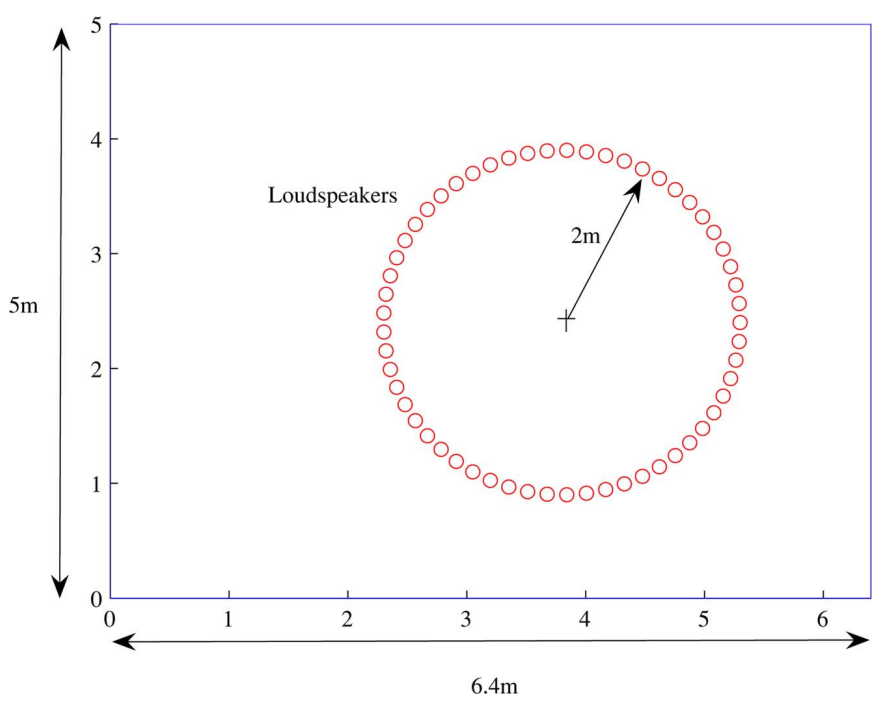

Fig. 6. Layout of the loudspeakers in the reverberant room. The room is rectangular with dimensions $6.4 \mathrm{~m} \times 5 \mathrm{~m}$ with wall absorption coefficient of 0.3 . We have equally placed 57 loudspeakers on a circle of $1.5 \mathrm{~m}$ centered about [3.8 $\mathrm{m}, 2.4 \mathrm{~m}]$

stration purpose, we only plot the actual reproduced field errors inside the zones of interest.

\section{B. Two-Zone Example in Reverberant Room}

In this example, we consider two circular reproduction zones of radii $0.5 \mathrm{~m}$ each which are in-line with each other in a reverberant room. As shown in Fig. 6, the room is rectangular with dimensions $6.4 \mathrm{~m} \times 5 \mathrm{~m}$ with wall absorption coefficient of 0.3 . We equally place 57 loudspeakers on a circle of $1.5 \mathrm{~m}$ centered at $[3.8 \mathrm{~m}, 2.4 \mathrm{~m}]$, which is our global origin $\mathcal{O}$ with respect to the bottom-left corner of the room. The reverberation is generated with a 2-D adaptation of the image-source method [15], [30], including image-sources up to the fifth order. Zone 1 and Zone 2 are both $1.0 \mathrm{~m}$ away from the global origin $\mathcal{O}$. The desired soundfields consist of 50 random plane waves at $1000 \mathrm{~Hz}$. We calculate loudspeaker weights using the reverberation design described in Section VI.

The resulting reproduced soundfield is shown in Fig. 7. The top-left plot (a) shows the real part of the desired soundfield, the top-right plot (b) shows the real part of the reproduced soundfield in a free field, the bottom-left plot (c) shows the real part of the same free field design in the reverberant room, and the bottom-right plot (d) shows the real part of the reproduced field with the reverberation design proposed in [15]. The reproduction errors calculated based on (24) in (b), (c), (d) are $0.59 \%$, $105 \%$ and $1.69 \%$, respectively.

\section{Reproduction Error}

1) Reproduction Error With Distance: We investigate the performance soundfield reproduction with two zones of radius $0.5 \mathrm{~m}$ each by plotting the mean squared reproduction error (24) with different zone separation in distance, i.e., $r^{(12)}$ (in meters), both in free field and in a reverberant room. These two reproduction zones are in-line with each other. The desired soundfields consist of 50 random plane waves at $1000 \mathrm{~Hz}$. We equally place 57 loudspeakers on a circle of $1.5 \mathrm{~m}$. The reverberation is 

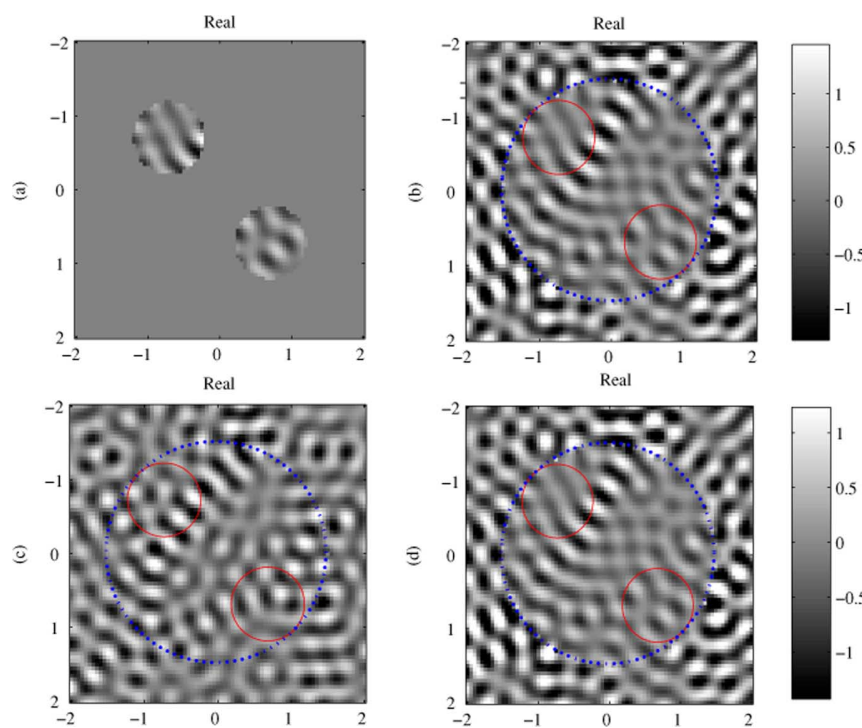

Fig. 7. Reproductions of a 2-D two-zone soundfield with radius $0.5 \mathrm{~m}$ each in free field and in a reverberant room. The desired soundfields consist of 50 random plane waves at $1000 \mathrm{~Hz}$. Two zones are located at $\theta^{(10)}=135^{\circ}, r^{(10)}=1 \mathrm{~m}$ and $\theta^{(20)}=-45^{\circ}, r^{(20)}=1 \mathrm{~m}$, respectively. (a) Desired field, (b) reproduced field in a free field, (c) the same free field design in the reverberant room, and (d) reproduced field with the reverberation design. We have equally 57 loudspeakers on a circle of $R=1.5 \mathrm{~m}$, where the loudspeaker positions are indicated in the dotted circle. The reproduction errors calculated based on (24) in (b), (c), and (d) are $0.59 \%, 105 \%$ and $1.69 \%$, respectively.

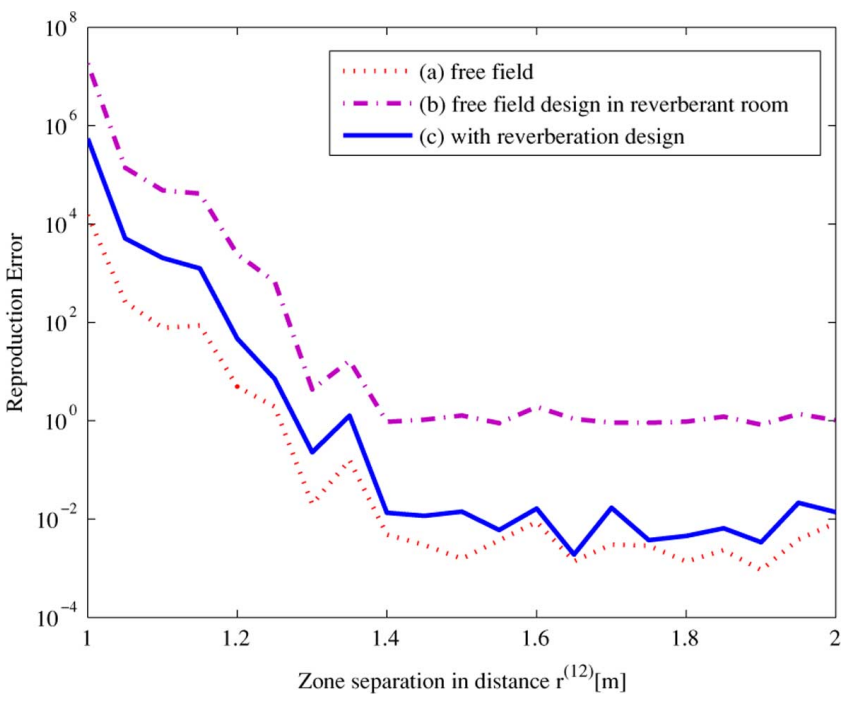

Fig. 8. Reproduction error (24) with different zone separation (in distance) $r^{(12)}(m)$ for two circular reproduction zones with radius $0.5 \mathrm{~m}$ each. These two reproduction zones are in-line with each other. The desired soundfields consist of 50 random plane waves at $1000 \mathrm{~Hz}$. We have equally placed 57 loudspeakers on a circle of $1.5 \mathrm{~m}$. (a) The reproduction errors in free field, (b) the reproduction errors with the same free field design in the reverberant room, and (c) the reproduction errors with the reverberation design.

generated according to the parameters set in Section VII-B. The result is shown in Fig. 8. We realize that the reproduction error decreases when zones are further apart. However, once $r^{(12)}$ is beyond a certain threshold, a larger zone separation will not increase the reproduction accuracy. The reproduction errors in reverberant case are consistent with the results obtained in free field.

2) Reproduction Error With Angle: We also investigate the reproduction error as a function of angle difference between

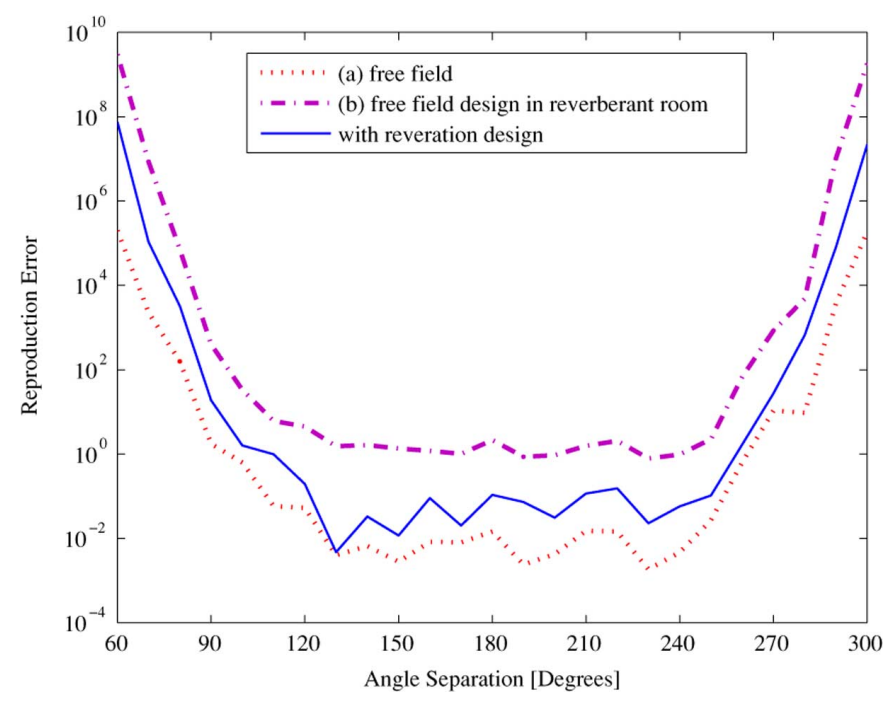

Fig. 9. Reproduction error (24) with different zone separation (in angles) $\theta^{(20)}-\theta^{(10)}$ for two circular reproduction zones with radius $0.5 \mathrm{~m}$ each. The desired soundfields consist of 50 random plane waves at $1000 \mathrm{~Hz}$. We have equally placed 57 loudspeakers on a circle of $1.5 \mathrm{~m}$. The angle separations are chosen as $60^{\circ}<\left(\theta^{(20)}-\theta^{(10)}\right)<300^{\circ}$ to provide two nonoverlapping soundfields. (a) The reproduction errors in free field, (b) the reproduction errors with the same free field design in the reverberant room, and (c) the reproduction errors with the reverberation design.

zone 2 and zone $1, \theta^{(20)}-\theta^{(10)}$, both in free field and reverberation rooms. Fig. 9 shows the reproduction errors for two circular reproduction zones with radius $0.5 \mathrm{~m}$ each. The desired soundfields consist of 50 random plane waves at 1000 $\mathrm{Hz} .57$ loudspeakers are equally placed on a circle of $1.5 \mathrm{~m}$. In order to recreate two nonoverlapping soundfields, we choose $60^{\circ}<\left(\theta^{(20)}-\theta^{(10)}\right)<300^{\circ}$. The large reproduction errors in the angle ranges $60^{\circ}$ to $90^{\circ}$ and $270^{\circ}$ to $300^{\circ}$ are mainly due to the poor conditioning of the matrix $\boldsymbol{T}(k)$, which has been discussed in the second comment in Section III. The consistent results hold for reverberation cases.

\section{Multizone Examples in Free Field}

In this example, we consider three circular reproduction zones with radius $0.5 \mathrm{~m}$ each in free field as shown in Fig. 10. We equally place 57 loudspeakers on a circle of 1.5 $\mathrm{m}$. Three zones are located at $\theta^{(10)}=45^{\circ}, r^{(10)}=1 \mathrm{~m}$, $\theta^{(20)}=165^{\circ}, r^{(20)}=1 \mathrm{~m}$, and $\theta^{(30)}=-75^{\circ}, r^{(30)}=1 \mathrm{~m}$, respectively. The desired soundfields consist of 50 random plane waves at $1000 \mathrm{~Hz}$. The top two plots show the real and imaginary parts of the desired soundfield, and the bottom two plots show the real and imaginary parts of the reproduced soundfield. The reproduction error calculated based on (24) is 9.85\%. Fig. 11 depicts the actual reproduced field error between the desired soundfields and the reproduced soundfields at each point inside the zones of interest (see (25) for definition). The ability to reproduce the three-zone soundfield using the same loudspeaker setting as that used in previous two-zone soundfield example validates guidelines proposed in Section V.

Fig. 12 shows the ability to reproduce a 2-D three-zone soundfield with different radii of $0.4,0.5$, and $0.6 \mathrm{~m}$, respectively, in free field. We equally place 57 loudspeakers on a circle of $1.5 \mathrm{~m}$. Three zones are located at $\theta^{(10)}=45^{\circ}, r^{(10)}=1.1$ $\mathrm{m}, \theta^{(20)}=165^{\circ}, r^{(20)}=1 \mathrm{~m}$, and $\theta^{(30)}=-75^{\circ}, r^{(30)}=0.9$ 

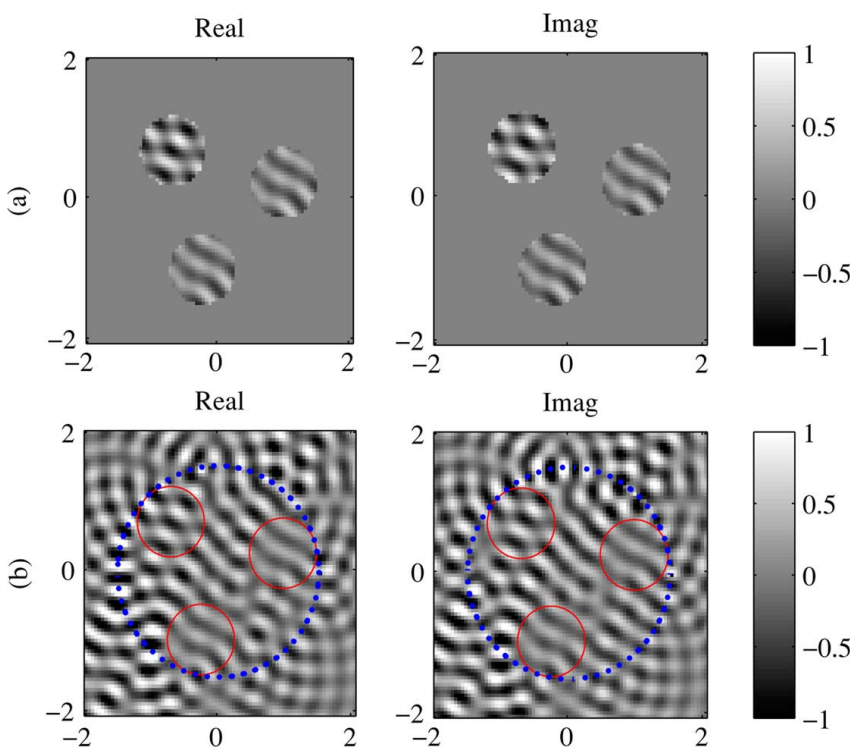

Fig. 10. Reproduction of a 2-D three-zone soundfield with radius $0.5 \mathrm{~m}$ each in free field. Three zones are located at $\theta^{(10)}=45^{\circ}, r^{(10)}=1 \mathrm{~m}, \theta^{(20)}=$ $165^{\circ}, r^{(20)}=1 \mathrm{~m}$, and $\theta^{(30)}=-75^{\circ}, r^{(30)}=1 \mathrm{~m}$, respectively. (a) Desired field, and (b) reproduced field. The desired soundfields consist of 50 random plane waves at $1000 \mathrm{~Hz}$. We have equally placed 57 loudspeakers on a circle of $1.5 \mathrm{~m}$, where the loudspeaker positions are indicated with the dotted circle. The reproduction error calculated based on (24) is $9.85 \%$.

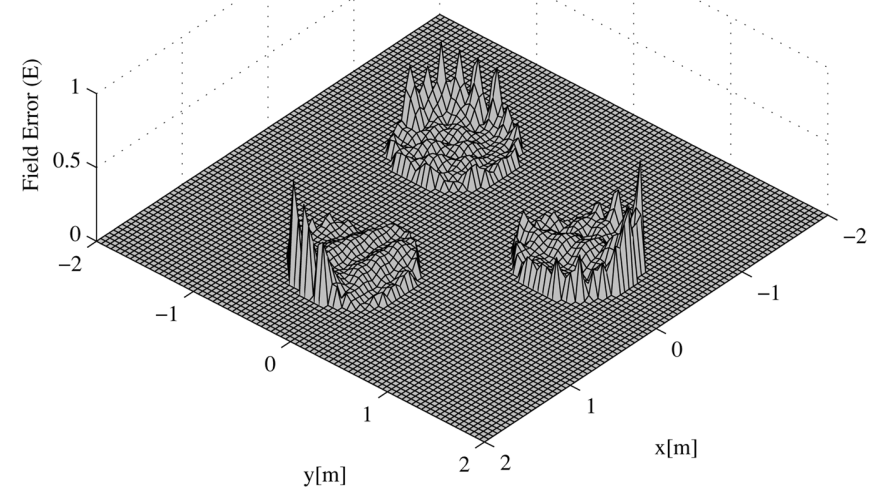

Fig. 11. Actual reproduced field error $\mathrm{E}\left(R^{(q)}, \Omega^{(q)} ; k\right)$ defined in (25) of a 2-D three-zone soundfield with radius $0.5 \mathrm{~m}$ each in free field. Three zones are located at $\theta^{(10)}=45^{\circ}, r^{(10)}=1 \mathrm{~m}, \theta^{(20)}=165^{\circ}, r^{(20)}=1 \mathrm{~m}$, and $\theta^{(30)}=-75^{\circ}, r^{(30)}=1 \mathrm{~m}$, respectively. The desired soundfields consist of 50 random plane waves at $1000 \mathrm{~Hz}$. We have equally placed 57 loudspeakers on a circle of $1.5 \mathrm{~m}$. For demonstration purpose, we only plot the actual reproduced field error (25) at each point in the zones of interest.

$\mathrm{m}$, respectively. The desired soundfields consist of 50 random plane waves at $1000 \mathrm{~Hz}$. The top two plots show the real and imaginary parts of the desired soundfield, and the bottom two plots show the real and imaginary parts of the reproduced soundfield. In this case, the reproduction error calculated based on (24) is $9.89 \%$. The actual reproduced error between the desired soundfields and the reproduced soundfields at each point inside the zones of interest (see (25) for definition) is plotted in Fig. 13. This simulation applied the same loudspeaker setting as used in previous examples and the promising result again validates the rule of thumb proposed in Section V.
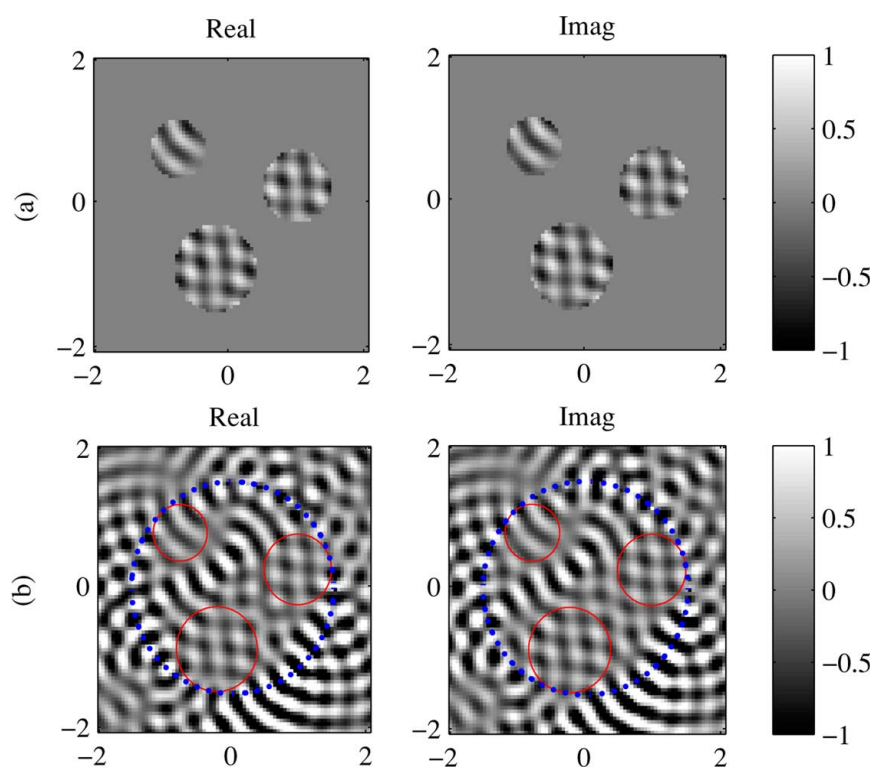

Fig. 12. Reproduction of a 2-D three-zone soundfield with different radii 0.4 $\mathrm{m}, 0.5 \mathrm{~m}, 0.6 \mathrm{~m}$ respectively in free field. Three zones are located at $\theta^{(10)}=$ $45^{\circ}, r^{(10)}=1.1 \mathrm{~m}, \theta^{(20)}=165^{\circ}, r^{(20)}=1 \mathrm{~m}$, and $\theta^{(30)}=-75^{\circ}, r^{(30)}=$ $0.9 \mathrm{~m}$, respectively. (a) Desired field, and (b) reproduced field. The desired soundfields consist of 50 random plane waves at $1000 \mathrm{~Hz}$. We have equally placed 57 loudspeakers on a circle of $1.5 \mathrm{~m}$, where the loudspeaker positions are indicated with the dotted circle. The reproduction error calculated based on (24) is $9.89 \%$.

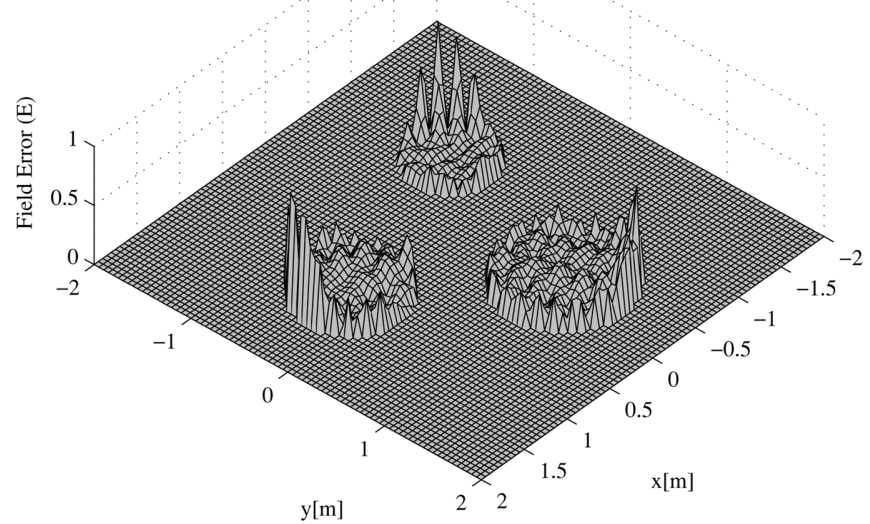

Fig. 13. Actual reproduced field error $\mathrm{E}\left(R^{(q)}, \Omega^{(q)} ; k\right)$ defined in $(25)$ of a 2-D three-zone soundfield with different radii $0.4 \mathrm{~m}, 0.5 \mathrm{~m}$, and $0.6 \mathrm{~m}$, respectively, in free field. Three zones are located at $\theta^{(10)}=45^{\circ}, r^{(10)}=1.1 \mathrm{~m}$, $\theta^{(20)}=165^{\circ}, r^{(20)}=1 \mathrm{~m}$, and $\theta^{(30)}=-75^{\circ}, r^{(30)}=0.9 \mathrm{~m}$, respectively. The desired soundfields consist of 50 random plane waves at $1000 \mathrm{~Hz}$. We have equally placed 57 loudspeakers on a circle of $1.5 \mathrm{~m}$, where the loudspeaker positions are indicated with the dotted circle. For demonstration purpose, we only plot the actual reproduced field error (25) at each point in the zones of interest.

\section{CONCLUSION}

In this paper, we provide a framework to recreate a 2-D multizone soundfield using a single array of loudspeakers by cylindrical harmonic expansions. We presented a novel approach of finding the equivalent global soundfield coefficients by the spatial harmonic coefficients translation theorem. This approach makes full use of the available modes and generates more modes freedom. Quantitative performances of a 2-D soundfield reproduction system have been provided with two-zone examples and 
multizone examples in both free field and reverberant environments. Reproduction errors are investigated in terms of distance separations and angle separations. Some fundamental limits of 2-D multizone soundfield reproduction were also revealed. The number of zones could be reproduced inside the single circular array was determined by the total number of modes from each zone, which was proportional to the size of the region. The extensions of the multizone soundfield reproduction design in reverberant rooms were shown in this paper.

\section{APPENDIX I}

\section{A. Proof of Theorem 1}

The soundfield at a point $\left(r^{(2)}, \theta^{(2)}\right)$ with respect to the origin $\mathrm{O}_{2}$ (see Fig. 3) can be written as

$$
S\left(r^{(2)}, \theta^{(2)} ; k\right)=\sum_{n=-\infty}^{\infty} \alpha_{n}^{(2)}(k) J_{n}\left(k r^{(2)}\right) e^{i n \theta^{(2)}}
$$

where $\alpha_{n}^{(2)}(k)$ are the soundfield coefficients with respect to the origin $\mathcal{O}_{2}$.

From Graf's addition formula [31]-[33], we write

$$
\begin{aligned}
J_{n}\left(k r^{(2)}\right) e^{j n \theta^{(2)}}=\sum_{m=-\infty}^{\infty} & T_{m}^{(21)}\left(r^{(12)}, \theta^{(12)} ; k\right) \\
& \times J_{n+m}\left(k r^{(1)}\right) e^{i(m+n) \theta^{(1)}}
\end{aligned}
$$

where $T_{m}^{(21)}\left(r^{(12)}, \theta^{(12)} ; k\right) \triangleq J_{m}\left(k r^{(12)}\right) e^{-j m \theta^{(12)}}$. By substituting (27) in (26), we obtain

$$
\begin{aligned}
S\left(r^{(2)}, \theta^{(2)} ; k\right) & =\sum_{n=-\infty}^{\infty} \sum_{m=-\infty}^{\infty} \alpha_{n}^{(2)}(k) T_{m}^{(21)} \\
& \times\left(r^{(12)}, \theta^{(12)} ; k\right) J_{n+m}\left(k r^{(1)}\right) e^{i(m+n) \theta^{(1)}} .
\end{aligned}
$$

By a change of variable $m+n=\ell$, we have

$$
\begin{aligned}
S\left(r^{(2)}, \theta^{(2)} ; k\right)= & \sum_{m=-\infty}^{\infty} \sum_{\ell=-\infty}^{\infty} \alpha_{\ell-m}^{(2)}(k) T_{m}^{(21)} \\
& \times\left(r^{(12)}, \theta^{(12)} ; k\right) J_{\ell}\left(k r^{(1)}\right) e^{i \ell \theta^{(1)}} .
\end{aligned}
$$

Since $S\left(r^{(1)}, \theta^{(1)} ; k\right) \equiv S\left(r^{(2)}, \theta^{(2)} ; k\right)$,

$$
\begin{aligned}
S & \left(r^{(1)}, \theta^{(1)} ; k\right) \\
= & \sum_{\ell=-\infty}^{\infty} \alpha_{\ell}^{(1)}(k) J_{\ell}\left(k r^{(1)}\right) e^{i \ell \theta^{(1)}} \\
= & \sum_{\ell=-\infty}^{\infty}\left[\sum_{m=-\infty}^{\infty} \alpha_{\ell-m}^{(2)}(k) T_{m}^{(21)}\left(r^{(12)}, \theta^{(12)} ; k\right)\right] \\
& \times J_{\ell}\left(k r^{(1)}\right) e^{i \ell \theta^{(1)}}
\end{aligned}
$$

where $\alpha_{\ell}^{(1)}(k)$ are the coefficients of the soundfield with respect to the origin $\mathcal{O}_{1}$. Thus,

$$
\begin{aligned}
\alpha_{\ell}^{(1)}(k) & =\sum_{m=-\infty}^{\infty} \alpha_{\ell-m}^{(2)}(k) T_{m}^{(21)}\left(r^{(12)}, \theta^{(12)} ; k\right) \\
& =\alpha_{\ell}^{(2)}(k) * T_{\ell}^{(21)}\left(r^{(12)}, \theta^{(12)} ; k\right)
\end{aligned}
$$

which completes the proof.

\section{B. Proof of Theorem 2}

From the Translation Theorem 1 and the fact $\theta^{(21)}=\theta^{(12)}+$ $\pi$, we can write

$$
\begin{aligned}
T_{m}^{(12)}\left(r^{(21)}, \theta^{(21)}\right) & =J_{m}\left(k r_{0}\right) e^{-i m \theta^{(21)}} \\
& =J_{m}\left(k r_{0}\right) e^{-i m\left(\theta^{(12)}+\pi\right)} \\
& =T_{m}^{(21)}\left(r^{(12)}, \theta^{(12)}+\pi\right) .
\end{aligned}
$$

\section{REFERENCES}

[1] M. A. Gerzon, "Periphony: With-height sound field reproduction," $J$. Audio Eng. Soc., vol. 21, pp. 2-10, Jan. 1973.

[2] J. S. Bamford, "An analysis of ambisonics sound systems of first and second order," Ph.D. dissertation, M.Sc. thesis, Université de Waterloo, Waterloo, ON, Canada, Jul. 1995.

[3] J. Daniel, R. Nicol, and S. Moreau, "Further inverstigations of high order ambisonics and wave field synthesis for holophonic sound imaging," in Proc. 114th Audio Eng. Soc. (AES) Conv., Amsterdam, The Nertherlands, Mar. 2003.

[4] M. Poletti, "Robust two-dimensional surround sound reproduction for nonuniform loudspeaker layouts," J. Audio Eng. Soc., vol. 55, no. 7/8, pp. 598-610, July/Aug. 2007.

[5] O. Kirkeby and P. A. Nelson, "Reproduction of plane wave sound fields," J. Acoust. Soc. Amer., vol. 94, no. 5, pp. 2992-3000, Nov. 1993.

[6] O. Kirkeby, P. A. Nelson, F. Orduna-Bustamante, and H. Hamada, "Local sound field reproduction using digital signal processing," $J$. Acoust. Soc. Amer., vol. 100, no. 3, pp. 1584-1593, Sept. 1996.

[7] M. Poletti, "Three-dimensional surround sound systems based on spherical harmonics," J. Audio Eng. Soc., vol. 53, no. 11, pp. 1004-1025, Nov. 2005.

[8] A. J. Berkhout, "A holographic approach to acoustic control," J. Audio Eng. Soc., vol. 36, pp. 977-955, Dec. 1988.

[9] A. J. Berkhout, D. de Vries, and P. Vogel, "Acoustic control by wave sound field synthesis," J. Acoust. Soc. Amer., vol. 93, pp. 2764-2778, May 1993.

[10] D. de Vries, "Sound reinforcement by wave field synthesis: Adaptation of the synthesis operator to the loudspeaker directivity characteristics," J. Audio Eng. Soc., vol. 44, no. 12, pp. 1120-1131, Dec. 1996.

[11] D. de Vries and M. M. Boone, "Wave field synthesis and analysis using array technology," in Proc. IEEE Workshop Applicat. Signal Process. Audio Acoust. (WASPAA'99), New Paltz, NY, Oct. 1999, pp. 15-18.

[12] M. M. Boone, E. N. G. Verheijen, and P. F. van Tol, "Spatial soundfield reproduction by wave-field synthesis," J. Audio Eng. Soc., vol. 43, no. 12, pp. 1003-1012, Dec. 1995.

[13] S. Spors, R. Rabenstein, and J. Ahrens, "The theory of wave field synthesis revisited," in Proc. 124th Audio Eng. Soc. (AES) Conv., Amsterdam, The Netherlands, May 2008.

[14] D. B. Ward and T. D. Abhayapala, "Reproduction of plane wave sound field using an array of loudspeakers," IEEE Trans. Speech Audio Process., vol. 9, no. 6, pp. 697-707, Sep. 2001.

[15] T. Betlehem and T. D. Abhayapala, "Theory and design of sound field reproduction in reverberant rooms," J. Acoust. Soc. Amer, vol. 117, no. 4, pp. 2100-2111, Apr. 2005.

[16] Y. J. Wu and T. D. Abhayapala, "Soundfield reproduction using theoretical continuous loudspeaker," in Proc. IEEE Int. Conf. Acoust, Speech, Signal Process. (ICASSP'08), Las Vegas, NV, Mar. 2008, pp. $377-380$.

[17] Y. J. Wu and T. D. Abhayapala, "Theory and design of soundfield reproduction using continuous loudspeaker concept," IEEE Trans. Speech, Audio, Lang. Process., vol. 17, no. 1, pp. 107-116, Jan. 2009.

[18] "Microsoft's gadgets of the future get their day in the limelight," Mar. 2007. 
[19] I. Tashev, "Personal Audio Space." [Online]. Available: http://research. microsoft.com/en-us/people/ivantash/ 2007

[20] Computer Speakers for Your Ears Only Mar. 2007 [Online]. Available: http://www.technologyreview.com/Infotech/18413/?a=f, Mar. 21, 2007, MIT Technology Review

[21] M. Poletti, "An investigation of 2-D multizone surround sound systems," in Proc. 125th Audio Eng. Soc. (AES) Conv., San Francisco, CA, Oct. 2008.

[22] Y. J. Wu and T. D. Abhayapala, "Spatial multizone soundfield reproduction," in Proc. IEEE Int. Conf. Acoust, Speech, Signal Process. (ICASSP'09), Taipei, Taiwan, Apr. 2009, pp. 93-96.

[23] Y. J. Wu and T. D. Abhayapala, "Multizone 2-D soundfield reproduction via spatial band stop filters," in Proc. IEEE Workshop Applicat. Signal Process. Audio Acoust. (WASPAA'09), New Paltz, NY, Oct. 2009 , pp. 309-312.

[24] T. D. Abhayapala and Y. J. Wu, "Spatial soundfield reproduction with zones of quiet," in Proc. 127th Audio Eng. Soc. (AES) Conv., New York, Oct. 2009.

[25] R. A. Kennedy, P. Sadeghi, T. D. Abhayapala, and H. M. Jones, "Intrinsic limits of dimensionality and richness in random multipath fields," IEEE Trans. Signal Process., vol. 55, no. 6, pp. 2542-2556, Jun. 2007.

[26] H. M. Jones, R. A. Kennedy, and T. D. Abhayapala, "On dimensionality of multipath fields: Spatial extent and richness," in Proc. IEEE Int. Conf. Acoust., Speech, Signal Process. (ICASSP'02), Orlando, FL, May 2002, vol. 3, pp. 2837-2840.

[27] J. Ahrens and S. Spors, "An analytical approach to sound field reproduction with a movable sweet spot using circular distributions of loudspeakers," in Proc. IEEE Int. Conf. Acoust, Speech, Signal Process. (ICASSP'09), Taipei, Taiwan, Apr. 2009, pp. 273-276.

[28] J. Ahrens and S. Spors, "Spatial encoding and decoding of focused virtual sound sources," in Proc. Ambisonic Symp., Graz, Austria, Jun. 2009.

[29] F. M. Fazi and P. A. Nelson, "The ill-conditioning problem in sound field reconstruction," in Proc. 123rd Audio Eng. Soc. (AES) Conv., Madrid, Spain, Oct. 2007.

[30] J. B. Allen and D. A. Berkley, "Image method for efficiently simulating small-room acoustics," J. Acoust. Soc. Amer., vol. 65, pp. 943-950, 1979.

[31] More Properties of Hankel and Bessel Functions. Columbus: The Ohio State Univ. [Online]. Available: http://www.math.ohio-state.edu/ gerlach/math/BVtypset/node127.html
[32] M. Abramowitz and I. A. Stegun, Handbook of Mathematical Functions. New York: Dover, 1972.

[33] , J. A. Stratton, Ed., Electromagnetic Theory. New York: McGrawHill, 1941.

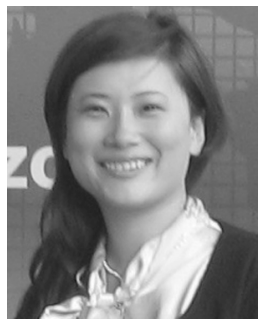

Yan Jennifer Wu received the B.E. degree (with honors) in electrical engineering and the M.E. degree in engineering form the University of Queensland, Brisbane, Australia, and the Ph.D. degree in engineering from the Australian National University (ANU), Canberra, Australia in 2010.

Her research interests are in the multichannel sound reproduction systems, array signal processing, and physical layer wireless communication systems.

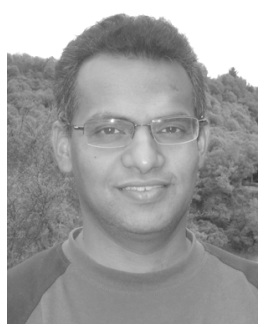

Thushara D. Abhayapala (M'00-SM'08) received the B.E. degree (with honors) in interdisciplinary systems engineering and the $\mathrm{Ph}$.D. degree in telecommunications engineering, from the Australian National University (ANU), Canberra, in 1994 and 1999, respectively.

From 1995 to 1997, he was a Research Engineer with the Arthur C. Clarke Center for Modern Technologies, Sri Lanka. Since December 1999, he has been a faculty member at ANU. He was the Leader of the Wireless Signal Processing (WSP) Program at the National ICT Australia (NICTA) from November 2005 to June 2007. Currently, he is the head of the school of Engineering at the Australian National University. His research interests are in the areas of spatial audio and acoustic signal processing, space-time signal processing for wireless communication systems, and array signal processing. He has supervised 24 research students and coauthored over 160 peer-reviewed papers. He is currently an Associate Editor for the EURASIP Journal on Wireless Communications and Networking.

Dr. Abhayapala is a Member of the Audio and Acoustic Signal Processing Technical Committee (2011-2013) of the IEEE Signal Processing Society. 